\title{
Effect of melatonin on oxidative stress markers in patients with alopecia areata
}

\author{
AL- Gaff AN*, Humadi S.*, Wohaieb S.A,** \\ * Departments of clinical pharmacy \& pharmacology, College of pharmacy, University of Baghdad \\ **Ministry of health, (Alkarama hospital), Baghdad, Iraq
}

Received: 1/11/4...; Accepted: 11/0/r..0

Alopecia areata is an autoimmune, nonscarring, multifocal disorder of hair of the growth characterized by circular bald areas, which occur on any hair bearing site body. The exact cause of alopecia areata is unknown. Recent study reported a potential role of oxygen free radicals (OFR) in pathogenesis of alopecia areata.

This clinical study was designed to evaluate the antioxidant effect of melatonin ( $\sim \mathrm{mg}$ every other day ) in patients with alopecia areata. This study revealed that two month treatment with melatonin antioxidant led to a decrease in the basal level of malondialdehyde (MDA) an index of lipid peroxidation, an increase in basal glutathione (GSH) content a major endogenous antioxidant in both plasma and erythrocytes and an increase in plasma total antioxidant status( TAS ). The most important point is the clinical significance of antioxidants in improving the hair growth response of patients with alopecia areata. This may be due to direct and/or indirect effect of melatonin on immune system. The direct effect may be through its immunoenhancing / immunostimulatory properties of melatonin. The indirect effect is the scavenging activity of this antioxidant, which in turn decrease damaging effect of oxygen free radicals (OFR) and utilization of GSH in neutralizing phagocytesinduced free radicals. So replenishment of GSH within natural killer (immune) cells strengthens the immune system and increases the rate of hair growth.

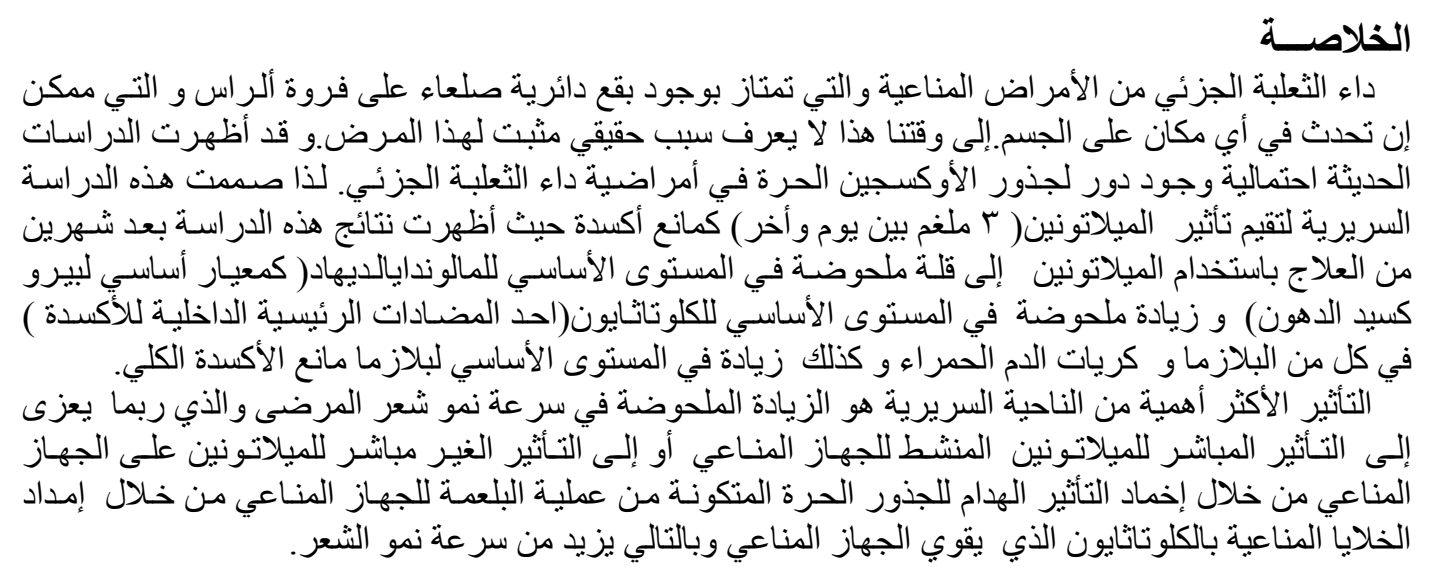

\section{Introduction}

A lopecia areata is a nonscarring hair-loss condition that often has a patchy distribution and can be quite unpredictable ${ }^{(1-r)}$. Alopecia areata is believed to be an autoimmune disease ${ }^{(\xi-}$
`) involving $\mathrm{T}$ lymphocytic infiltration around the hair follicle ${ }^{(v, \wedge)}$. Therapeutic options depend on several factors, including amount of hair-bearing skin involved and the age of the patient ${ }^{(9)}$ Intralesional steroids have been a 
mainstay of treatment. Topical steroids, anthralin, and minoxidil are additional therapeutic modalities that have been used with mixed success over the last several years ${ }^{(', r)}$.

Once the classic treatments of dermatologists are exhausted, a number of people turn to the use of alternative treatments which includes(Stress reduction, Aloe vera, Poison ivy, Sunlight, Heat treatment, Zinc supplements, Mustard seed/Capsicum poultice, Vitamin supplements/creams, Asprin poultice, Dimethylsulfoxide (DMSO), Homeopathic treatment, Herbs, Flax seed oil, linseed oil, fish oil). Some of these therapies are recommended by the more experimental dermatologists or by alternative practitioners. Usually very little is known about alternative treatments in relation to their effects on alopecia $\operatorname{areata}^{(\cdot)}$.

few years ago, several studies have presented several lines of evidence for the involvement of oxygen free radicals (OFRs) in the pathogenesis of alopecia areata. Al-Jaff et.al. ${ }^{(')}$ investigated the role of oxidative stress in patients with AA , the effect of nutrient antioxidants on tissue antioxidant status and the clinical picture of the disease. Therapy with nutrient antioxidants in these patients significantly improved tissue antioxidant status and was associated with good responce in their hair growth .These effects suggest an important role of nutrient antioxidants in protecting the body (e.g immune system) from oxidative damage and may influence the severity of alopecia areata.

Melatonin is the principal hormone produced by the pineal gland (the base of the brain)that decreases with age ${ }^{(1 r, i r)}$ .The pineal gland monitors changing light levels throughout the day and, sensing the approach of nightfall, increases its output of melatonin. Nighttime melatonin levels are $1 \cdot$ times higher than daytime levels ${ }^{(T, / r)}$.It is the most powerful antioxidant known. It protects cells from free radical damage and delays the aging process ${ }^{\left(1 r_{-} / 0\right)}$. Melatonin also has immunostimulatory/ immunoenhancing properties ${ }^{(1 \%-10)}$. These studies have demonstrated a stimulatory effect of melatonin on acquired immunity, and reported to increase natural killer cell activity. A high affinity receptor binding site for melatonin has been found on $\mathrm{T}$ helper lymphocyte cells providing a direct link between melatonin levels and immunosensitivity. Melatonin activates these $\mathrm{T}$ lymphocytes into production of cytokines and this cascade of chemical signals recruits other immune cells and makes them more responsive ${ }^{(T)}$. One of the key targets for melatonin is the thymus as a central organ of the immune system.

Therefore, an oxidative stress may play an important role in hair loss in patients with alopecia areata, this clinical study was aimed at evaluating the antioxidant effect of melatonin and the degree of its effects on the levels of oxidative stress markers in patients with

alopecia areata.

\section{Subjects and methods Subjects}

A-study group: comprised of total of 1 . subjects, ${ }^{\circ}$ normal controls (mean age rr.A. $\pm r .99$ years) and 0 cases with alopecia areata(mean age ro.A. \pm r.) years). They were non-smokers, nonalcoholics and free from apparent other diseases.Patients involved in this study were under a dermatologist supervision and received a traditional treatment which includes [corticosteroids, diuretics (spironolactone), local irritant, phototherapy and tonics]. The duration of disease ranged from ( $r$ month -10 years). B-patients: five patients ( ) female, \& males) with alopecia areata were included in this study. Treatment schedules included a combination of antioxidants ( melatonin capsule $r \mathrm{mg}$ each other day) with traditional treatments which includes [corticosteroids (prednisolone tablet $10 \mathrm{mg} /$ day 
and intra-lesional corticosteroids), diuretics (spironolactone ro $\mathrm{mg}$ /day), local irritant, phototherapy and tonics like B- complex capsule]. The treatment with melatonin continued for two months.

C-Samples: heparinized venous blood samples were collected from alopecic patients as well as from controls using plastic disposable syringes. Fresh blood samples were used for MDA and GSH measurements in plasma and RBCs . Measurement of plasma and erythrocytes MDA based on the reaction of thiobarbituric acid (TBA) forming TBA-MDA adduct, was carried out using the modified method of Stocks and Dormandy ${ }^{(17)}$ as described by Gilbert et $\mathrm{al}^{\left({ }^{(\vee)}\right.}$. The results were expressed as $\mathrm{OD} / \mathrm{ml}$ and $\mathrm{OD} / \mathrm{g} \mathrm{Hb}$ respectively . Measurement of plasma and erythrocytes GSH content was determined to the method of Godin et al. ${ }^{(\curlywedge)}$. Amounts of GSH were expressed as $\mathrm{OD} / \mathrm{ml}$ and $\mathrm{OD} / \mathrm{g} \mathrm{Hb}$ respectively. Plasma samples were frozen up to $1 \varepsilon$ days for total antioxidant status measurement (TAS) using commercial assay kit obtained from Randox( $(19)$. Data were analyzed using t-test.
The baseline mean of plasma and erythrocytes MDA concentrations in patients with alopecia areata was significantly higher than those of controls. Treatment with the antioxidant melatonin normalized MDA levels in plasma and RBCs after $r$ months after treatment (Table 1).

Basal level of plasma GSH concentrations in patients with alopecia areata were significantly higher than those of controls . Treatment with melatonin did not normalize these levels, but rather had a significant effect in further increasing plasma GSH levels.

Furthermore, the basal mean RBCs GSH and plasma TAS levels were significantly lower in patients with alopecia areata than those of controls. Treatment with melatonin did significantly elevate GSH content and TAS levels to normal level in patients with alopecia areata after two months of treatment (Table I).

Clinically, there was an obvious improvement in rate of hair growth after 1 and $r$ months of treatment with the combination of the melatonin and traditional treatment as shown in the following pictures .

\section{Results}

Table( (1): Markers of oxidative stress in patients with alopecia areata with their aged matched controls before and after two months treatment with a r mg

melatonin each other day.

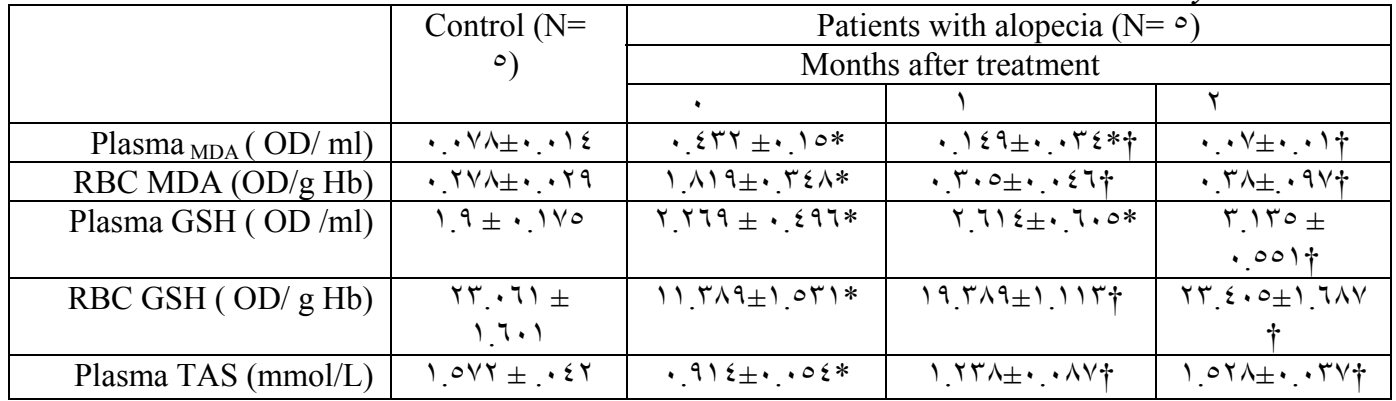

Values are expressed as mean \pm standared deviation.

*significantly different from control $(\mathrm{P}<. \cdots 0), \dagger$ significantly different from pretreatment $(\mathrm{P}<\because \cdots 0)$, MDA: malondialdehyde( an index of lipid peroxidation),

GSH: glutathione, TAS: total antioxidant status, RBC: erythrocytes 


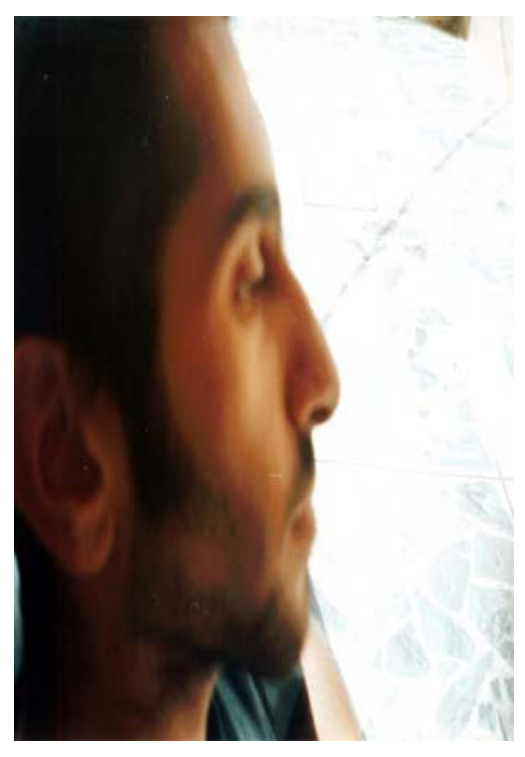

(A)

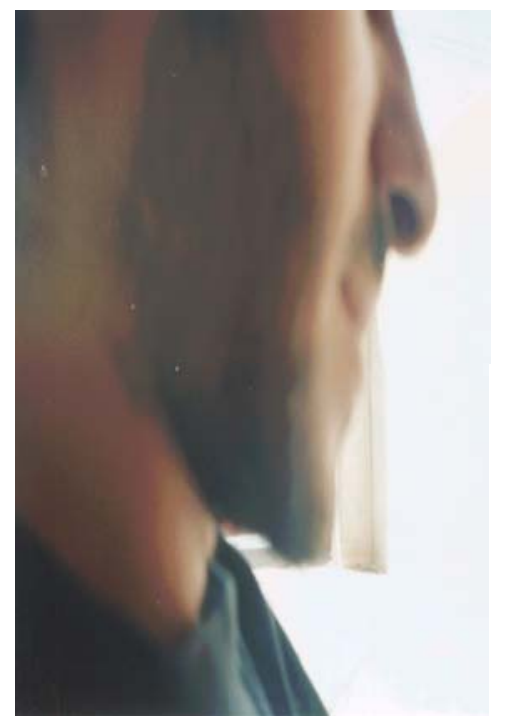

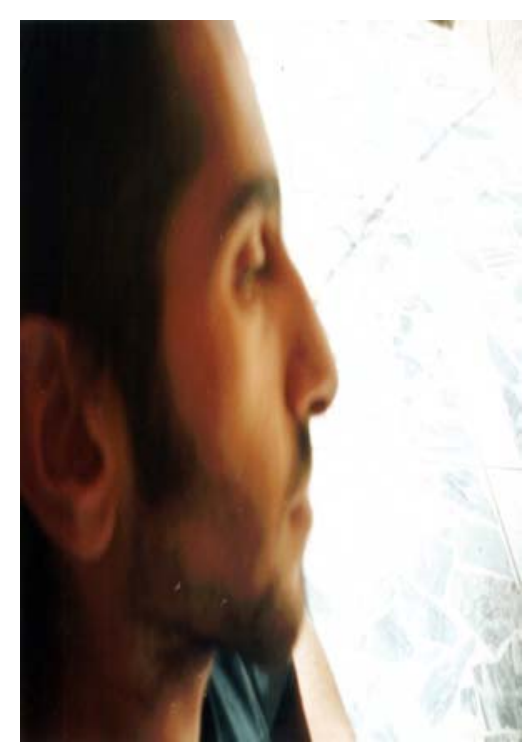

(B)

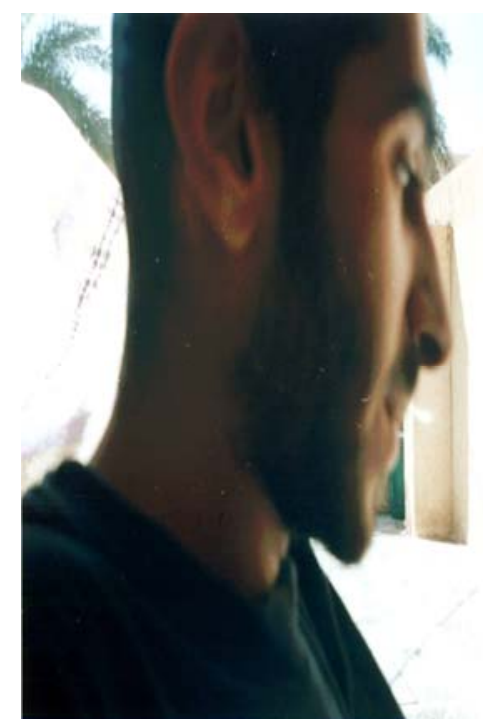

(C)

Patient with alopecia areata taking melatonin capsule ( $r \mathrm{mg})$ every other day : A,B before treatment, $\mathrm{C}$ during treatment and $\mathrm{D}$ after $r$ months of treatment.

Discussion

Various studies in humans and animals have shown that melatonin has a powerful pro-immune effect, especially in stressed, aging or diseased animals $^{(1 \leqslant, 10, r \cdot)}$. They noted that chronic melatonin treatment of old mice restores a number of age-associated immune alterations $^{(1 \leqslant, 10, r \cdot 9)}$. In particular, at the thymic level, regrowth of the organ and recovery of the reduced thymic endocrine activity, were also observed. At the peripheral level, corrections of the low number of peripheral blood lymphocytes and, at least, partial reconstitution of responsiveness to $\mathrm{T}$ cell mitogens are observed ${ }^{(r \cdot)}$.

The present study demonstrated, for the first time, the presence of 
[alterations in TAS].no comparable study is available in the literature in this regard and further studies are needed to investigate the mechanism (s) responsible for this finding. It seems alterations in oxygen free radical scavenging processes in erythrocytes and plasma of patients with alopecia areata manifested by a decrease in plasma antioxidant status (TAS) .It seems that antioxidant defenses remain high in normal individual, keeping lipid peroxidation under control. However, in alopecic patients antioxidant defenses are significantly lowered, thus exposing erythrocytes to the damaging effects of lipid peroxides. This may be due to a direct and/or indirect effect of alopecia, per se, where the already formed immune complex resulted in the production of phagocytes- derived free radicals, which will lead to the initiation of lipid peroxidation as manfisted by the significant increase in total endogenous basal MDA levels.

$\mathrm{GSH}$, a major scavenger of oxygen reactive intermediates, protect cells against the effect of free radical and of related intermediates (e.g. peroxides) that are formed endogenously ${ }^{(r)}$. Therefore, a reduction in cellular GSH may be associated with an increased susceptibility to oxidant stress or reflect a response to $i^{\left({ }^{(r)}\right)}$. A considerable decrease in the red cell GSH has already been reported in patients with alopecia $^{(r)}$. In the present study, the decreased total GSH content in red cells may be due to the increased GSH utilization in neutralizing oxygen free radical produced by phagocytes. These changes in GSH level may increase the susceptibility of erythrocytes and plasma to endogenous oxidative stress. The fact that plasma GSH level of alopecic patients were higher than their controls may be a compensatory increase due to the release of oxidized GSH from tissues and pooled in plasma. The treatment with melatonin antioxidant led to decrease the basal level of MDA increase GSH in both plasma and erythrocytes, and increase plasma TAS.Furthermore, the most important clinical effect of melatonin combined with traditional treatment, in improving the hair growth responce of alopecic patients, has not been previously reported in the available litrature. This interesting and noval finding may be due to direct and/or indirect effects of melatonin. The direct effect include its immunostimulatory and immunoenhancing effects ${ }^{\left(\Upsilon \xi, r^{\circ}\right)}$ which is particularly apparent in immunodepressive state.The immunoenhancing action of melatonin seems to be mediated by T-helper cellderived opioid peptides as well as by lymphokines and perhaps by pituitary hormones. Melatonin- inducedimmuno-opioids and lymphokines imply the presence of specific binding sites or melatonin receptors on cells on immune system.On the other hand, lymphokines such as gamma-interferon and interleukin-r as well as thymic hormones can modulate the synthesis of melatonin in the pineal gland ${ }^{(\Upsilon)}$. The indirect effect of melatonin include its scavenging activity, which in turn decrease both oxidative damage and utilization of GSH in neutralizing phagocytes induced free radicals. So replenishment of GSH within natural killer (immune) cells strengthens the immune system and increases the rate of hair growth. In conclusion, the present study further supports the role of oxidative stress in the pathogenesis of alopecia areata, and presents a novel evidence for the preferential effect of the powerful antioxidant and immunostimulating hormone, mela-tonin when combined with traditional treatment in correcting anti-oxidant status as well as improving the rate of hair growth in alopecia areata patients in comparison with the effects obtained from the use of traditional trea-tment alone.Further studies are required to invistigate the 
exact mechanism responsible for this novel effect of melatonin.

1. Thomas P References Dermatology" A color guide to diagnosis and therapy, $\varepsilon^{\text {th }}$ edition, Mobsy,Philadelphia,Pennsylvania. ${ }^{\top}$.. $\varepsilon ; P^{\wedge} 00$.

r. Champio-RH; Burton-JL; BurnsDA;Breathnach,Eds. Rook" Textbook of dermatology" ${ }^{\text {th }}$ edition, Blackwell science,England.r..r;':prq.r_rqr^.

r. Fauci-A.S.,Braunwald- E, isselbacher- K. J, Harrisons" Principles of internal Medicine" Eds. s $^{\text {th }}$ edition, Mc Graw-Hill,New York. Y.. ';prIr. ए)

£. Barahamani N; De Andrade M,Slusser J;Zhang Q;Duvic M. Interleukin- $)$ receptor antagonist allele $r$ and familial alopecia areata.

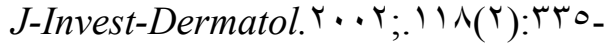
$\mathrm{v}$.

๑. Tazi-Ahini R; Cork MJ;Gawkrodger D J;et al. Role of the autoimmune regulator (AIRE) gene in alopecia areata : strong association of a potentially functional AIRE polymorphism with alopecia universalis. Tissue antigens. $r . . r$; 7.(7): $\left\{\wedge 9 \_90\right.$.

7. Madani S,and Shapiro J. Alopecia areata update. J Am Acad Dermatol. r...; ; r:0 : 9_0 Tr.

$\checkmark$. Freyschmidt-Paul P, Ziegler A, McElwee KJ, et al. Treatment of alopecia areata in $\mathrm{C}^{\mathrm{r}} \mathrm{H} / \mathrm{HeJ}$ mice with the topical immunosuppressant FK 0.7 (Tacrolimus). Eur J Dermatol. r.. I; 11: $: 0 . \varepsilon \cdot 9$.

^. Amos Gilhar. Mediation of alopecia areata by co- operation between $\mathrm{CD}^{2}$ and $\mathrm{CD}^{\wedge+} \mathrm{T}$

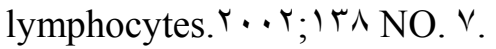

१. Papadopoulos AJ, Schwartz RA, and Janniger CK. Alopecia areata. Pathogenesis, diagnosis, and therapy. Am J Clin Dermatol. Y...;1:1,1 - 1.0.

1. Alopecia areata: alternative treatment (Keralin.com) internet.
1). AL- Jaff A.The role of oxidative stress in alopecia areata. M.SC. thesis. University of Baghdad $Y .$. l.

Ir. Beard H.O. social and psychological implication of alopecia areata.J-AmAcad-Dermatol. 19人7;) ₹:79v.

1T. Maestroni GJ.The immunotherapeutic potential for melatonin.Expert Opin Investig

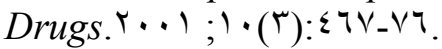

$1 \leq$. Reiter RJ. Areview of the evidence supporting melatonins role as an antioxidant.J Pineal Res. 1990 Jan; ' $(1): 1-11$.

10. Reiter RJ. Melatonin as free radical scavenger : implications for a ging and age related disease.Ann NY Acad Sci.199ะ, r1;V19:1-1r.

17. Stocks-J and Dormandy-T.Lthe autooxidation of human red blood cell lipids induced hydroge peroxidee.Brit-J-

Hematol. $\left.19 \vee 1 ; r \cdot: 90_{-}\right) 11$.

IV. Gilbert-HS;Stump-DD and RothEF.Amethod to correct for errors caused by generation of interfering compounds during erythrocyte lipid peroxidation.Anal-

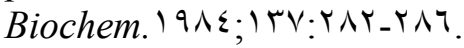

1^. Godin-DV;Wohaib-SA;Garnett-

ME.,and Goumeiouk-AD.Antioxidant enzyme alterations in expermental and clinical diabetes.Mole Cellular-

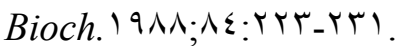

19. Miller NJ;Rice-Evance C;Duvies MJ;Gopinathan V.and Milner A.Total antioxidant status by colometric method.Clinical

Science. $199 \mathrm{r}_{;} \wedge \varepsilon: \varepsilon \cdot \nu_{-} \varepsilon 1 \mathrm{r}$.

$r \cdot$. Maestroni GJM,Conti A and Lissoni P: Type $r$ Th cells as target of the circadian melatonin signal:Relevance in local immunity. Regional Immuno$\operatorname{logy}(U S A) .1990 ; 710_{-}$( (ro._ros).

$r$ ). Meister-A.Selective modification of glutathione etabolism.Science. $19 \wedge \mathrm{r}$; YY.: $\leqslant Y Y_{-}-\mathrm{Y}$.

$r$ r. Chance-B;Sies-H;Boveris-A. Hydroperoxide metabolism in mamalian

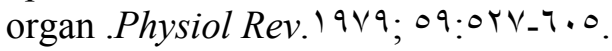


$r$. Kobrinsky-NL,Hartfield D,Horner

$\mathrm{H}$,et al.Treatment of advanced malignancies with high dose acetaminophen and $\mathrm{N}$-acetylcystene rescue.Cancer-Invest. $1997 ; 1 \leqslant(r)$ :

$\left.r \cdot r_{-}\right)$.

Y . Maestroni GJM, and Conti A: The pineal hormone melatonin stimulates activated $\mathrm{CD}^{\varepsilon+}, \mathrm{Th}^{-1}+$ cells to release opiod a gonist(s) with immunoenhancing and anti-stress properties.J Neuroimmunol (Netherlands). 199.; YN(r): 1 TV_V .

ro. Poon AM,Lin ZM, Pang CS,et al. Evidence for direct action of melatonin on immune system.Biol Signals(Switzerland). $199 \leqslant ; \Gamma(\Upsilon)$ : $1 \cdot V_{-}$ IV.

r7. Maestroni GJ.The immunoneuroendocrine role of melatonin. $J$ pineal Research (Denemark). 1994;। \&(1): 1-1. 
